Claremont Colleges

Scholarship@ Claremont

All HMC Faculty Publications and Research

HMC Faculty Scholarship

6-1-2007

\title{
Hot Electron and X-ray Production from Intense Laser Irradiation of Wavelength-Scale Polystyrene Spheres
}

\author{
H. A. Sumeruk \\ University of Texas at Austin \\ S. Kneip \\ University of Texas at Austin \\ D. R. Symes \\ University of Texas at Austin \\ I. V. Churina \\ University of Texas at Austin \\ A. V. Belolipetski \\ University of Texas at Austin \\ See next page for additional authors
}

\section{Recommended Citation}

H. A. Sumeruk, S. Kneip, D. R. Symes, I. V. Churina, A. V. Belolipetski, G. Dyer, J. Landry, G. Bansal, A. Bernstein, T. D. Donnelly, A. Karmakar, A. Pukhov and T. Ditmire, "Hot electron and x-ray production from intense laser irradiation of wavelength-scale polystyrene spheres," Phys. Plasmas, 14, 062704 (2007). doi: 10.1063/1.2746024 in All HMC Faculty Publications and Research by an authorized administrator of Scholarship @ Claremont. For more information, please contact scholarship@cuc.claremont.edu. 


\section{Authors}

H. A. Sumeruk, S. Kneip, D. R. Symes, I. V. Churina, A. V. Belolipetski, G. Dyer, J. Landry, G. Bansal, A. C. Bernstein, Thomas D. Donnelly, A. Karmakar, A. Pukhov, and T. Ditmire 


\section{Physics of}

\section{Hot electron and x-ray production from intense laser irradiation of wavelength-scale polystyrene spheres}

H. A. Sumeruk, S. Kneip, D. R. Symes, I. V. Churina, A. V. Belolipetski et al.

Citation: Phys. Plasmas 14, 062704 (2007); doi: 10.1063/1.2746024

View online: http://dx.doi.org/10.1063/1.2746024

View Table of Contents: http://pop.aip.org/resource/1/PHPAEN/v14/i6

Published by the AIP Publishing LLC.

\section{Additional information on Phys. Plasmas}

Journal Homepage: http://pop.aip.org/

Journal Information: http://pop.aip.org/about/about_the_journal

Top downloads: http://pop.aip.org/features/most_downloaded

Information for Authors: http://pop.aip.org/authors

\section{ADVERTISEMENT}

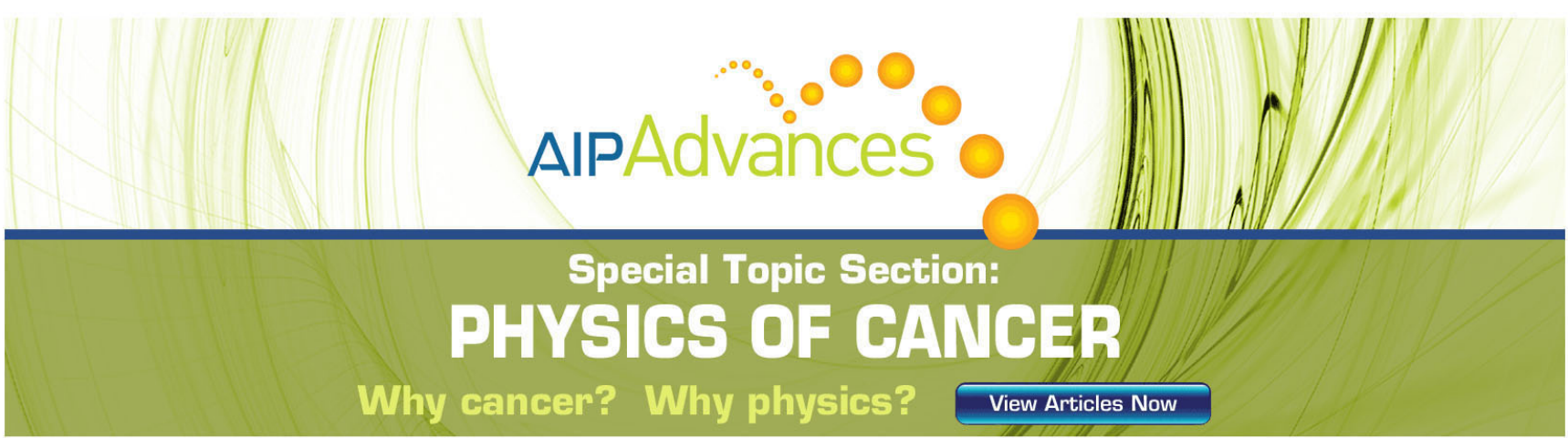




\title{
Hot electron and $x$-ray production from intense laser irradiation of wavelength-scale polystyrene spheres
}

\author{
H. A. Sumeruk, S. Kneip, D. R. Symes, I. V. Churina, A. V. Belolipetski, G. Dyer, \\ J. Landry, G. Bansal, and A. Bernstein \\ The Texas Center for High Intensity Laser Science, Department of Physics, The University of Texas, \\ Austin, Texas 78712 \\ T. D. Donnelly \\ Department of Physics, Harvey Mudd College, Claremont, California 91711
}

\author{
A. Karmakar and A. Pukhov \\ Institut fuer Theoretische Physik I, Heinrich-Heine-University of Dusseldorf, 40225 Dusseldorf, Germany
}

T. Ditmire

The Texas Center for High Intensity Laser Science, Department of Physics, The University of Texas, Austin, Texas 78712

(Received 9 March 2007; accepted 10 May 2007; published online 25 June 2007)

\begin{abstract}
Hot electron and $\mathrm{x}$-ray production from solid targets coated with polystyrene-spheres which are irradiated with high-contrast, $100 \mathrm{fs}, 400 \mathrm{~nm}$ light pulses at intensity up to $2 \times 10^{17} \mathrm{~W} / \mathrm{cm}^{2}$ have been studied. The peak hard $\mathrm{X}$-ray signal from uncoated fused silica targets is an order of magnitude smaller than the signal from targets coated with submicron sized spheres. The temperature of the $\mathrm{x}$-rays in the case of sphere-coated targets is twice as hot as that of uncoated glass. A sphere-size scan of the x-ray yield and observation of a peak in both the x-ray production and temperature at a sphere diameter of $0.26 \mu \mathrm{m}$, indicate that these results are consistent with Mie enhancements of the laser field at the sphere surface and multipass stochastic heating of the hot electrons in the oscillating laser field. These results also match well with particle-in-cell simulations of the interaction. (C) 2007 American Institute of Physics. [DOI: 10.1063/1.2746024]
\end{abstract}

\section{BACKGROUND}

Ultraintense laser-matter interactions with solids have attracted attention as a method for the production of bright, ultrafast incoherent $\mathrm{x}$-ray pulses. ${ }^{1,2}$ Interest in production of these short bursts of $\mathrm{x}$-rays is motivated by various possible applications including medical imaging and imaging of biological samples. An important advantage of the short pulse is that it generates a high $\mathrm{x}$-ray yield in a short time which can lead to reduced overall dosage. Other possible applications include the ultrafast probing of atomic structure whereby one could "film" the intermediate processes of a chemical reaction. Employed in combination with large scale implosion machines short pulse laser driven hard x-ray sources can be used to take high temporal and spatial resolution radiographs of hydrodynamic behavior in inertial confinement fusion implosions or other high density hydrodynamic experiments. ${ }^{3}$ These x-ray sources result from the production of hot electrons ( $\mathrm{keV}$ to $\mathrm{MeV}$ energies) which produce bremsstrahlung photons or induce inner shell atomic processes in a solid to produce the X-ray pulse.

To generate efficiently such bright $\mathrm{x}$-rays, intense, short laser pulses $\left(<10^{-12} \mathrm{~s}\right)$ focused to intensity $>10^{17} \mathrm{~W} / \mathrm{cm}^{2}$ are required for the production of the high peak current electron bunches needed for X-ray conversion. Frequently the laser pulses should have high-contrast to prevent the formation of long, lower-density plasma pedestals so that the laser energy is deposited in a high-density plasma. The highdensity allows for rapid electron conduction of the thermal energy and facilitates fast expansion and cooling, thereby shortening the duration of the thermal plasma produced $\mathrm{x}$-ray pulse and optimizing conversion of laser energy to the hottest electrons. Some small prepulse is often helpful, however, in creating a small amount of plasma in front of the target to aid in electron acceleration. ${ }^{4}$

A major barrier to coupling laser energy into a dense plasma is that at these high densities the plasma acts as a fairly good mirror. Processes such as resonance absorption ${ }^{5}$ and vacuum heating ${ }^{6}$ only occur when the angle of incidence of the laser is away from normal and there is a component of the electric field in the direction of the density gradient. This necessitates an off-normal laser angle of incidence which lowers the effective intensity on the target. To circumvent this problem, structured surfaces were suggested as a way to reduce reflectivity and increase absorption.

Over the past decade several experiments have been conducted to study the production of x-rays using targets with surfaces structured at different spatial scales. Murnane et al. ${ }^{7}$ studied the effect of nanometer surface structuring on reflectivity and hence absorption into the target. The reflectivity of a flat surface was shown to be $90 \%$ at normal incidence. Replacing the plain target with a grating, or covering the surface with gold nanoclusters, reduced the reflectivity at high intensity to $10 \%$ and correspondingly the absorption rose from $10 \%$ in flat targets to $90 \%$ in their structured targets. In these experiments the periodicity of the gratings was smaller than the wavelength, at roughly a half and a third of the wavelength, and the gold clusters consisted of particles 
of roughly $5 \mathrm{~nm}$ size and an average density of $0.3 \%$ solid. These targets had a dramatic effect on the emission of thermal plasma generated soft $\mathrm{x}$-rays $(<1 \mathrm{keV})$. Gold clusters produced double the $\mathrm{x}$-ray conversion efficiency of the grating $(25 \%$ vs $12 \%)$ which was in turn much higher than a flat surface $(0.9 \%)$. However, the x-ray pulse produced by the gold clusters was longer than the one produced by the grating. These experiments showed that structuring the target increases the resistivity and hence the absorption. Gordon et $a l^{8}$ extended these early studies at lower intensity $\left(\sim 10^{16} \mathrm{~W} / \mathrm{cm}^{2}\right)$ to include laser intensities up to $10^{18} \mathrm{~W} / \mathrm{cm}^{2}$. They characterized the radiation as nonthermal with two components, one with a characteristic temperature of $700 \mathrm{eV}$ and the other at $3 \mathrm{keV}$, hinting at the importance of a hot electron component of the absorption. Kulcsar et al. ${ }^{9}$ carried out a similar study using nanostructured "velvet" consisting of 10-200 nm diameter, $1 \mu \mathrm{m}$ long metallic fibers protruding from a surface. In these experiments the laser wavelength was $1.054 \mu \mathrm{m}$, the pulse duration was $1 \mathrm{ps}$, and the intensity was $10^{17} \mathrm{~W} / \mathrm{cm}^{2}$. They demonstrated increased $\mathrm{x}$-ray production by a factor of 50 over a flat target in the soft $\mathrm{x}$-ray region of $\sim 125 \mathrm{eV}$.

These early experiments concentrated on the production of soft thermal x-rays or $K$-alpha emission below $2 \mathrm{keV}$. More recently, hard $\mathrm{x}$-ray $(>2-10 \mathrm{keV})$ production from laser irradiated structured targets has come under study. Rajeev et al. ${ }^{10}$ used a $100 \mathrm{fs}, 806 \mathrm{~nm}$ laser with an intensity of $10^{15}-10^{16} \mathrm{~W} / \mathrm{cm}^{2}$ and demonstrated that a copper surface which was roughened with average features of approximately $0.1 \mu \mathrm{m}$ size increased the yield of hard $\mathrm{x}$-rays (in the 30-120 keV photon energy range) by a factor of 4 over similar copper targets that have been polished. This increase of $x$-ray yield occurred without a significant change in effective $\mathrm{x}$-ray temperature. This group also examined $\mathrm{x}$-ray production $^{11}$ when they irradiated a copper target covered with copper nanoparticles via a sputtering technique. They created both spherical and elliptical particles of approximately $15 \mathrm{~nm}$ size. Elliptical particles produced 13 times the yield produced by a flat surface, and spherical particles produced only 3 times the yield. They attributed this difference to a "lightning-rod" effect and plasmon resonance in the elliptical targets. The lightning rod effect refers simply to the increased electric field found at the vicinity of sharp objects. The plasmon resonance of the particles was shown to peak at certain ratios of the major to minor axis of the elliptical particles. In another paper ${ }^{12}$ this group studied the effect of surface-roughness spatial scale on x-ray yield. They observed that increasing roughness size increases the $\mathrm{x}$-ray yield. They also conducted an angular scan of the $\mathrm{x}$-rays and found that the enhancement over a flat surface at angle of maximum yield $\left(35^{\circ}-45^{\circ}\right)$ is a factor of 5 . They attribute their signal to resonant absorption and the higher energy component to vacuum heating. More recently Gavrilov et $a l .{ }^{13}$ demonstrated an increase in yield and temperature of hot electrons for flat targets which had been modified with three different methods: etching pyramidal cavities, craters from laser ablation, and a laser induced grating. The $\mathrm{x}$-ray temperatures observed were several $\mathrm{keV}$ and temperatures roughly doubled when compared to the flat surface measurements.

Most of these studies have been carried out with targets in which the structures are a few nanometers and are, therefore, much smaller than the wavelength of light. It is well known that particles with sizes comparable to the light wavelength can lead to very large enhancements of the laser field $(\sim 10-100$ times the vacuum field for conducting particles) through so-called Mie resonances. ${ }^{14}$ Some work toward understanding intense laser interactions with micronscale particles has already been conducted. ${ }^{15-20}$ For example, experiments conducted by Donnelly et al. demonstrated that increased $\mathrm{x}$-ray temperatures were obtained by irradiating wavelength scale droplets when compared to planar slabs. ${ }^{15}$ Particle-in-cell simulations of these experiments suggested that not only were field enhancements important but that the heating of electrons that can occur as they pass back and forth through the particle when driven by the oscillating field also plays an important role. Another experiment, undertaken by Symes et al., demonstrated an anisotropy in the energy of ions generated from intense irradiation of wavelength scale droplets. ${ }^{21}$ The explanation for the anisotropy was that Mie enhancements in the field surrounding the sphere caused anisotropic heating. However, in both of these experiments a target was employed that was composed of a broad range of particle sizes, so the effects of Mie resonances for particular particle sizes could not be manipulated or observed.

In this paper we report on a set of experiments in which an intense, high contrast $400 \mathrm{~nm}$ pulse with under $100 \mathrm{fs}$ duration irradiates arrays of polystyrene particles of single, well-defined sizes deposited on the surface of a planar solid. Unlike previous studies, this approach has allowed systematic study of structure size effects on field enhancements and consequent hot electron generation. We were specifically interested in determining how the Mie enhancements affected hot electron and $\mathrm{x}$-ray production and so measured $\mathrm{x}$-ray signals as a function of sphere size. To achieve this, we irradiated polystyrene spheres of wavelength scale particles that were uniform in size and available in a selection of sizes. We examined hard x-rays with photon energy $>10 \mathrm{keV}$. By varying the size of these particles we find that an optimum particle size leads to enhanced hot electron production and higher electron temperature. The effect can, in some cases, lead to x-ray yield enhancements of over two orders of magnitude. We attribute this effect to electric field enhancements at the surface of the particle and multiple transit stochastic heating of the electrons.

\section{EXPERIMENTAL SETUP AND DETAILS}

The schematic of the experiment described here is illustrated in Fig. 1. We irradiate targets with the THOR laser at the University of Texas, a high intensity Ti:sapphire laser operating at a central wavelength of $800 \mathrm{~nm}$ and a pulse duration of $35 \mathrm{fs}^{22}$ At full energy, it is capable of delivering $0.7 \mathrm{~J}$ on target at $10 \mathrm{~Hz}$. For reasons explained below we frequency-doubled a portion of the laser with a $50 \mathrm{~mm}$ diameter, $2 \mathrm{~mm}$ thick potassium dihydrogen phosphate (KDP) crystal, and were able to deliver $10 \mathrm{~mJ}$ of $400 \mathrm{~nm}$ light on 


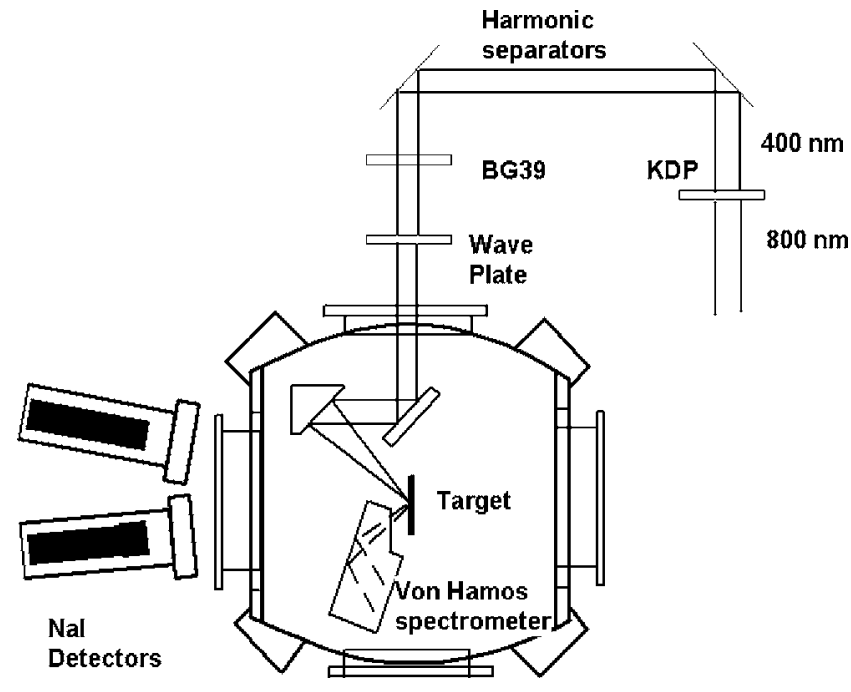

FIG. 1. Experimental set up for irradiation of sphere monolayers with $400 \mathrm{~nm}$ intense pulses. The $800 \mathrm{~nm}$ pulse is frequency doubled in the KDP crystal and then focused to high-intensity using the parabolic mirror. The BG39 absorbs residual $800 \mathrm{~nm}$ light remaining after the KDP crystal. NaI detectors are used to measure the $\mathrm{x}$-ray signal.

target with a pulse duration of $100 \mathrm{fs}$. The beam was reflected off of two dichroic mirrors to remove any residual $800 \mathrm{~nm}$ light. Using an $\mathrm{f} / 2.8$ parabolic mirror the $400 \mathrm{~nm}$ light was focused to a peak intensity of $2 \times 10^{17} \mathrm{~W} / \mathrm{cm}^{2}$ in a $6 \mu \mathrm{m}, 1 / e^{2}$-diameter focus. The laser frequency was doubled to avoid the generation of a plasma gradient at the target surface from prepulses (discussed below).

To explore the effects of microsphere size on the strong field interactions with hot electrons, we desired targets with single layers of nearly monodisperse spherical particles deposited on a polished substrate. After extensive trials, we found that the best avenue to achieving such a target was through the deposition of polystyrene spheres on thin smooth targets. In particular, we ultimately fielded targets of polished fused silica (and in some cases, not discussed in this paper), single crystal silicon slides which were coated with polystyrene spheres. The spheres used were delivered suspended in water in monodisperse solutions from Duke Scientific with diameter variations of $3 \%-5 \%$ for each size. The fused silica slides were found to be the optimum choice of substrates after lengthy trial and error experimentation; a critical factor in determining the deposition quality on the substrate is the surface roughness and the surface adsorption to the liquid used to suspend the spheres. Fused silica slides provided us
TABLE I. Dilution rations for various sphere sizes.

\begin{tabular}{ll}
\hline \hline Sphere size $(\mu \mathrm{m}$ diameter $)$ & Dilution ratio (ethanol:sphere solution) \\
\hline 0.1 & $50: 1$ \\
0.26 & $8.33: 1$ \\
0.36 & $5.55: 1$ \\
0.5 & $4.17: 1$ \\
0.74 & $4: 1$ \\
1.0 & $2: 1$ \\
2.9 & $2: 1$ \\
\hline
\end{tabular}

with an inexpensive substrate which we found allowed good deposition uniformity and sphere adherence. In addition, we imaged through the glass which proved valuable in aligning the sample before each shot.

The silica slides were cleaned with a $2 \%$ Hellmanex solution for $15 \mathrm{~min}$ in an ultrasonic bath and rinsed in Millipore water; this process was repeated 3 times. We deposited spheres by placing a $20 \mu \mathrm{l}$ drop of the sphere solution on an inclined surface at $9^{\circ}$ inclination which was protected from air currents. This technique was adapted from that of Micheletto et $a .^{23}$ We modified the technique described in Ref. 11 by replacing the diluting agent used in that paper with ethanol. This provided faster drying time and produced reasonable coverage without the need to have a finely controlled temperature. The optimum dilution ratio of the commercial sphere solution (which was typically about $10 \%$ polystyrene spheres by volume) with ethanol was found to vary with sphere size. We tabulate the optimum dilution ratio for each sphere size employed in Table I.

Each sphere size had its own coating characteristics but in general they could be categorized into two regimes. Spheres greater than $0.5 \mu \mathrm{m}$ diameter form patches of a hexagonally packed monolayers on the silica, illustrated in Fig. 2. Smaller spheres form a random monolayer of unconnected or multiply connected spheres which cover the surface with a density of 20\%-80\% for the various sizes, illustrated in Fig. 3 . For the larger spheres we used an imaging system which allowed us to position the laser focus either in the middle of a sphere patch or on bare silica.

To gain information about the hot electron production from irradiation of these targets, we collected data on the hard x-rays which are created by bremsstrahlung in the underlying substrate. If thin target bremsstrahlung is assumed, the x-ray temperature can be directly related to the hot elec-
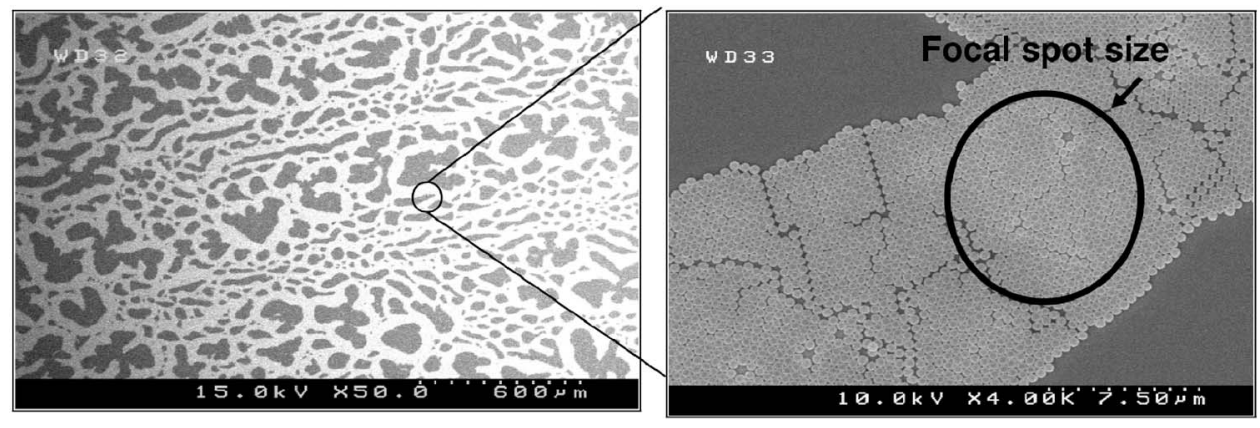

FIG. 2. A scanning electron microscope image of a target. Here, $0.5 \mu \mathrm{m}$ diameter spheres coat a glass-slide substrate in hexagonal close-pack patches. The laser focus is shown, and could be positioned to hit regions of either bare glass or sphere-coated glass as desired. 


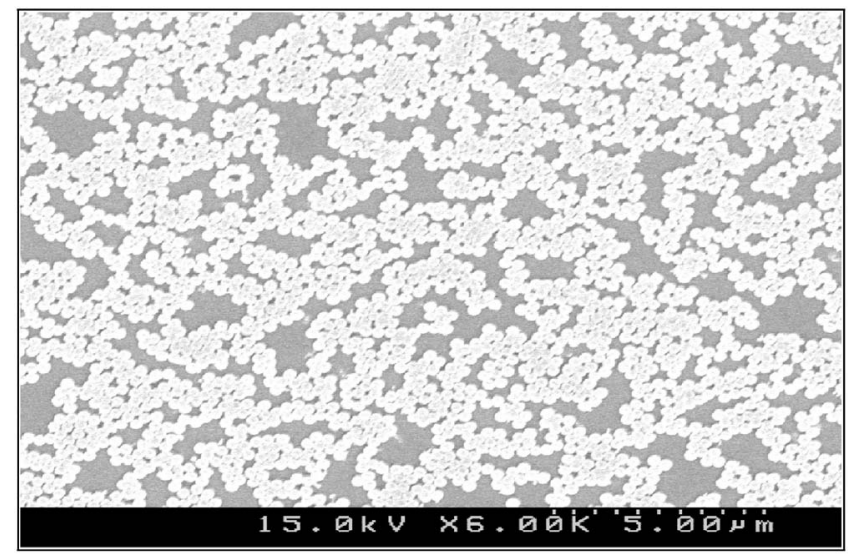

FIG. 3. A scanning electron microscope image of a target. Here, $0.26 \mu \mathrm{m}$ diameter spheres coat a silica-slide substrate in a random pattern.

tron temperature. ${ }^{24}$ To measure the $\mathrm{x}$-ray signals we employed six NaI detectors shielded in lead housing. All six detectors were placed at close proximity to each other in order to observe at a similar angle through a similar thickness of target chamber material, a Plexiglas vacuum flange. Each detector was filtered with slabs of different thicknesses of copper and stainless steel. The various combinations of filters allowed us to have photon cutoff energies of 23,31 , $40,52,65$, and $75 \mathrm{keV}$. The photon energy response of the detectors was calibrated using a multichannel analyzer and known radioactive sources.

\section{EXPERIMENTAL RESULTS}

With these targets, we first investigated irradiation with the fundamental wavelength of the laser at $800 \mathrm{~nm}$. We conducted trial runs of the experiment focusing a $15 \mathrm{~mJ}, 800 \mathrm{~nm}$ pulse to an intensity of $1 \times 10^{18} \mathrm{~W} / \mathrm{cm}^{2}$. In this configuration, however, a 24 ps prepulse at the $10^{-4}$ level was identified using a third-order autocorrelator. To study the effect of this prepulse we conducted an angle scan from which we plotted hard x-ray intensity from a single NaI detector versus angle of incidence using $p$-polarized irradiation on a planar silica target (uncoated with spheres). The results, shown in Fig. 4(a), were typical of resonant absorption with a maximum observed yield occurring at a $\sim 15^{\circ}$ incidence angle. Using the standard approximations for the scale length of optimum resonant absorption ${ }^{5}$ the plasma scale length, $\Delta L$, can be estimated. This scale length is $\Delta L \approx(c / \omega)$ $\times(0.8 / \sin \theta)^{3}$ for a laser of angular frequency, $\omega$, when the angle of maximum absorption is $\theta$. The data of Fig. 4(a) indicate a plasma scale length of $\sim 3.5 \mu \mathrm{m}$. This suggested that the small spheres would not survive the prepulse of the laser.

To clean this prepulse we chose to double the laser frequency in the KDP crystal and remove the residual $800 \mathrm{~nm}$ light, including the 24 ps prepulse, with dichroic mirrors. We repeated that the hard x-ray yield angle scan from an uncoated silica target with the $p$-polarized frequency-doubled pulse, and observed x-ray yield at photon energy $>20 \mathrm{keV}$. Those data are illustrated in Fig. 4(b). In this configuration the x-ray signal peaked near an incidence angle of $55^{\circ}$. When
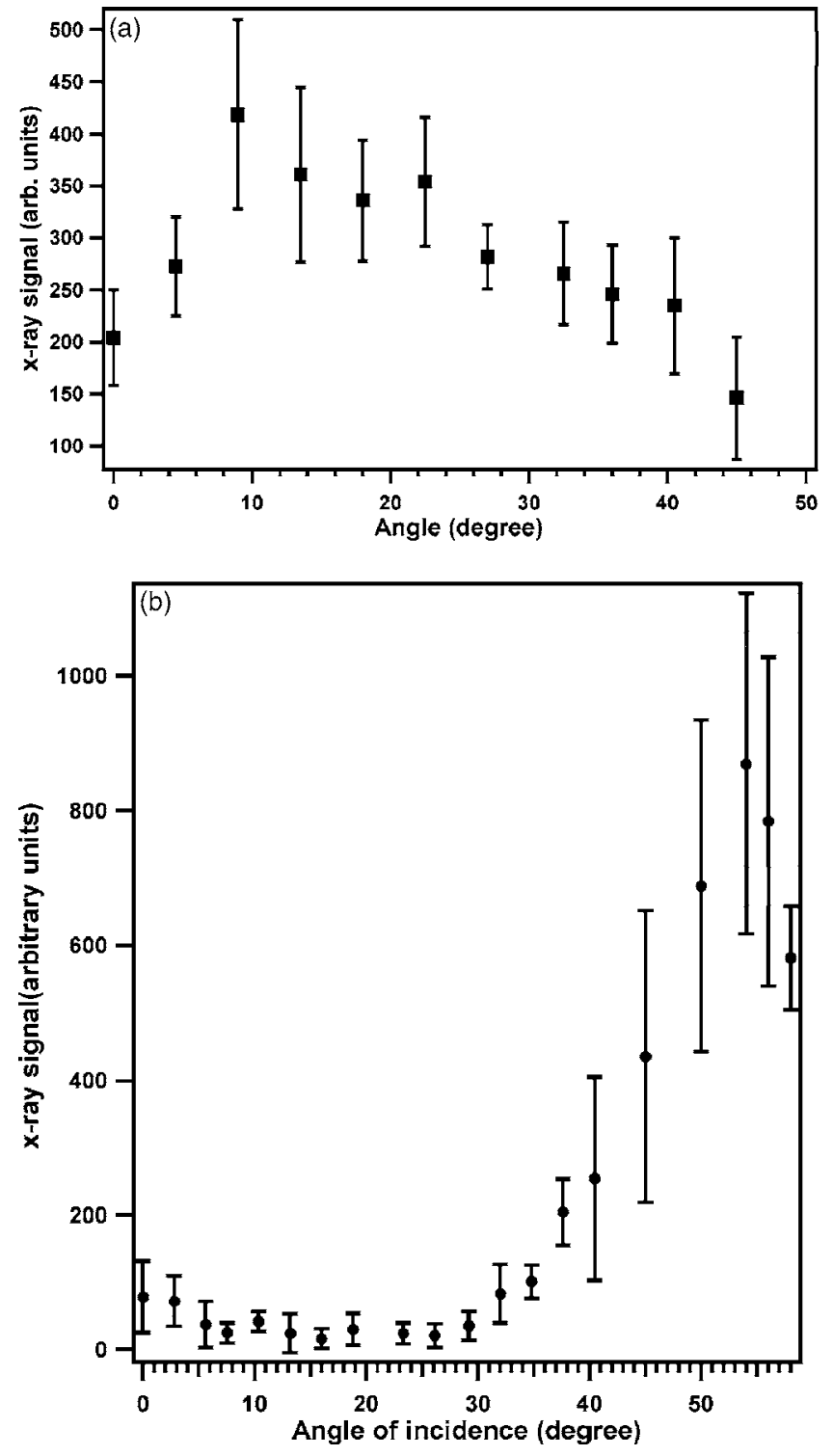

FIG. 4. Measured hard x-ray yield as a function of incident angle of the laser pulse when using (a) $800 \mathrm{~nm}$ light, or (b) $400 \mathrm{~nm}$ light to irradiate a planar silica slide. In (a) the yield is shown for x-rays above $600 \mathrm{keV}$ and the signal peaks near $15^{\circ}$, indicating that resonant absorption dominates the system. This absorption mechanism occurs for longer scale length plasmas and implies the presence of a significant laser prepulse. In (b) the yield is shown for $\mathrm{x}$-rays above $22 \mathrm{keV}$ and the signal peaks near $55^{\circ}$. This peak emission angle is significantly different than for the $800 \mathrm{~nm}$ light and indicates that the system is dominated by vacuum heating.

an intense $p$-polarized pulse interacts with a very sharp density gradient, less than an electron quiver amplitude, we expect that the hot electron production will be predominantly by vacuum heating, often termed Brunel heating. ${ }^{6}$ In this regime, hot electrons are pulled from the plasma by the field of the laser, accelerated in vacuum by the laser's field and returned to the plasma surface. The angle of maximum absorption in this case can be found by determining the angle for maximum perpendicular component of the laser electric field at the surface of the target. Since the intensity varies with the focal spot as $\cos \theta$, the perpendicular component of the laser field is $E_{\perp}=E_{0} \sin \theta \cos ^{1 / 2} \theta$ which is maximum at an angle of $55^{\circ}$. We, therefore, concluded that with $400 \mathrm{~nm}$ 


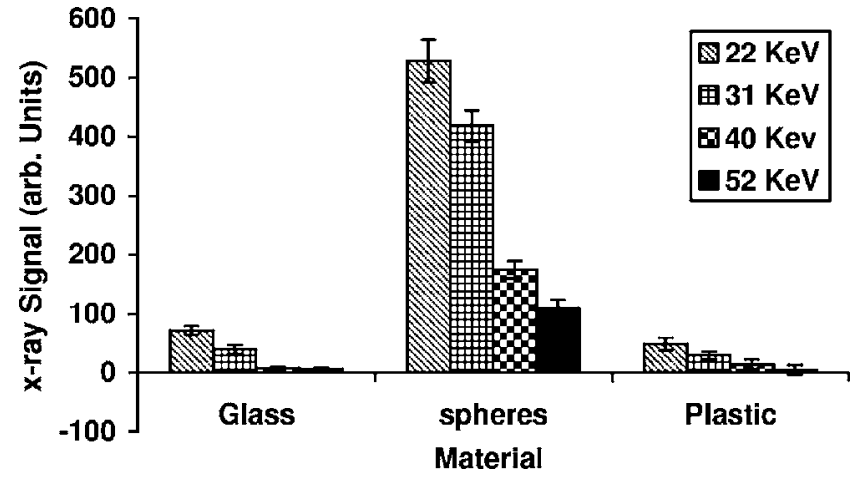

FIG. 5. X-ray yield comparison for three target materials. These data were collected with the laser at a $55^{\circ}$ angle of incidence. The signal from the sphere coated targets is an order of magnitude larger than either of the other target materials.

pulses at this intensity our interaction qualitatively corresponds to vacuum heating as modeled by Gibbon et al. ${ }^{25}$ This indicated that plasma expansion of well under the electron oscillation amplitude $\left(\lambda_{\text {osc }} \sim 15 \mathrm{~nm}\right)$ occurred and that our pulses would interact with well defined spheres that have not undergone significant expansion prior to the arrival of the peak of the pulse. Furthermore, we simulated the expansion of a spherical plasma using the Medusa 103 Lagrangian hydrodynamic code and confirmed that we expected very little expansion of these small spheres. ${ }^{26}$

We compared the $\mathrm{x}$-ray emission from an uncoated silica slide, a silica slide coated with a $0.3 \mu \mathrm{m}$ thick coating of poly(methyl methacrylate) (PMMA), similar in atomic composition to our polystyrene spheres, and a slide coated with $0.26 \mu \mathrm{m}$ diameter spheres. Comparative hard x-ray yields from these targets are shown in Fig. 5. At the incidence angle corresponding to the maximum $\mathrm{x}$-ray signal for planar silica targets $\left(55^{\circ}\right)$ we compared the $\mathrm{x}$-ray signal from the three target types: the signal from the sphere-covered silica was an order of magnitude larger than that of the uncoated silica, which in turn was slightly larger than that from the silica target with a thin PMMA layer. These data indicate that any enhancement that we observe from sphere coated targets cannot simply be attributed to the difference of the type of material on the target surface, which can modify the absorption properties, ${ }^{20}$ as the PMMA coated target exhibited yield almost identical to the uncoated silica, while a sphere coated target had a much higher $\mathrm{x}$-ray yield than either.

We measured hard x-ray yield from targets coated with spheres of seven different diameters. We deposited 0.100 , $0.260,0.360,0.500,0.744,1.00$, and $2.90 \mu \mathrm{m}$ diameter spheres on silica and took 25-30 laser shots at each size in a single experimental run. These runs were conducted at a normal incidence. At this incidence angle we observed almost no measurable hard $\mathrm{x}$-ray signal from planar silica targets. The measured hard $\mathrm{x}$-ray yield at photon energies greater than $22 \mathrm{keV}$ and $75 \mathrm{keV}$ as a function of sphere size is illustrated in Fig. 6. We observe a strong dependence of hard $\mathrm{X}$-ray production with the sphere size employed. We observed a clear peak in x-ray production when the $0.260 \mu \mathrm{m}$ spheres are employed. When compared to planar slabs this sphere size gives an enhancement in $\mathrm{x}$-ray yields of $>1000$.

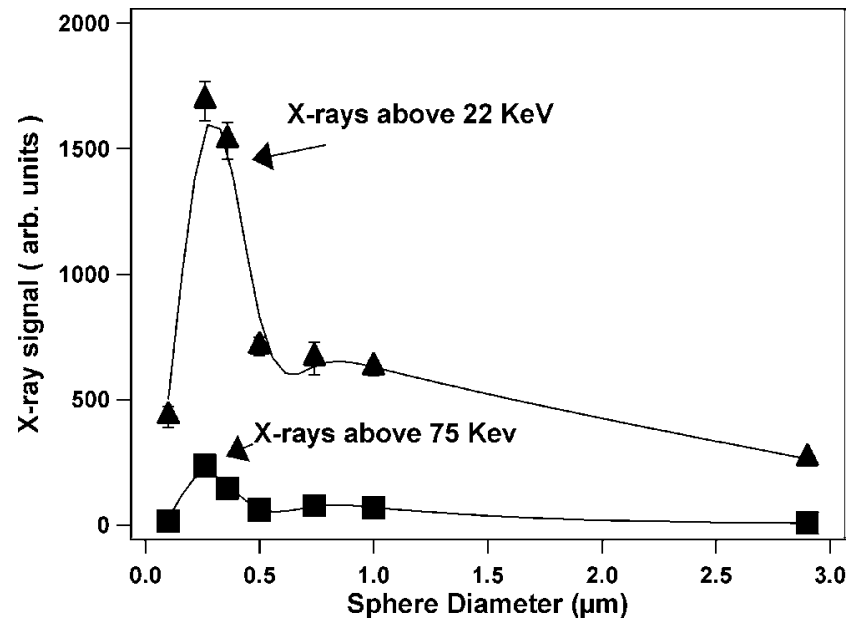

FIG. 6. Measured x-ray signal as a function of sphere diameter for two different cutoff filters. The lines through the data points are present to guide the eye. A pronounced peak in X-ray signal (through both filters) is evident near $0.26 \mu \mathrm{m}$.

Furthermore, we observed an enhancement by a factor of 8 in yield of x-rays $>22 \mathrm{keV}$ from targets coated by $260 \mathrm{~nm}$ spheres when compared to the yield from planar targets irradiated at $55^{\circ}$ incidence with $p$-polarization.

At each sphere size, we measured $x$-ray yield through all six x-ray filters. This allowed us to determine the hot electron temperature. To determine the temperature of hot electrons generated we fit an electron energy distribution to the data using the methodology described by McCall et al. ${ }^{27}$ Because we examine hard x-rays being emitted from a thin target, we can assume there is an exponential distribution of the bremsstrahlung $\mathrm{x}$-ray radiation and that the x-ray temperature and the hot-electron temperature are equivalent. ${ }^{25}$ The X-ray spectrum is then convolved with the detector response and filter transmission functions. We then found the hot-electron temperature which minimized the error in fit between the data and the implied exponential x-ray spectrum. Figure 7 plots $x$-ray yield measured through filters with various $1 / e^{2}$ cutoff energies from targets coated with

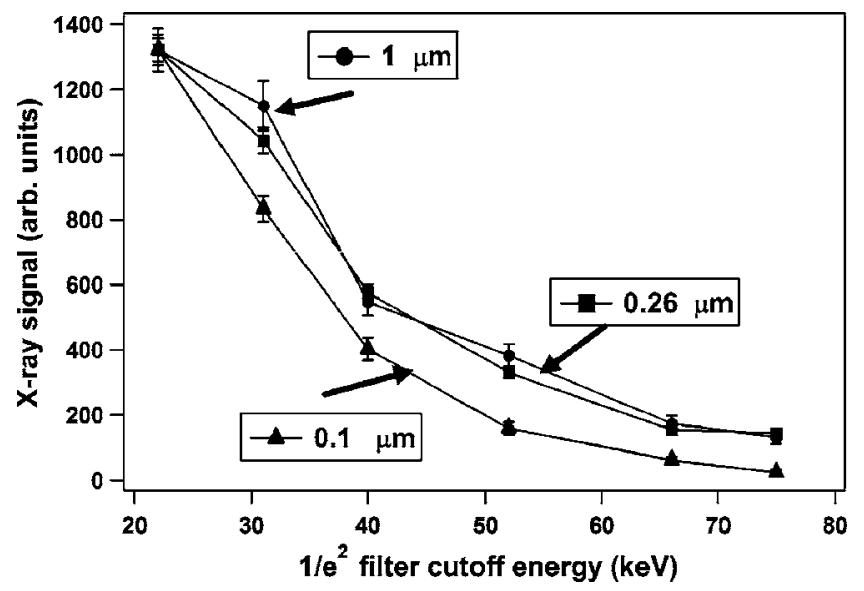

FIG. 7. Measured x-ray signal through different filter materials plotted as a function of the cutoff transmission photon energy. Data for three different sphere sizes are shown. 


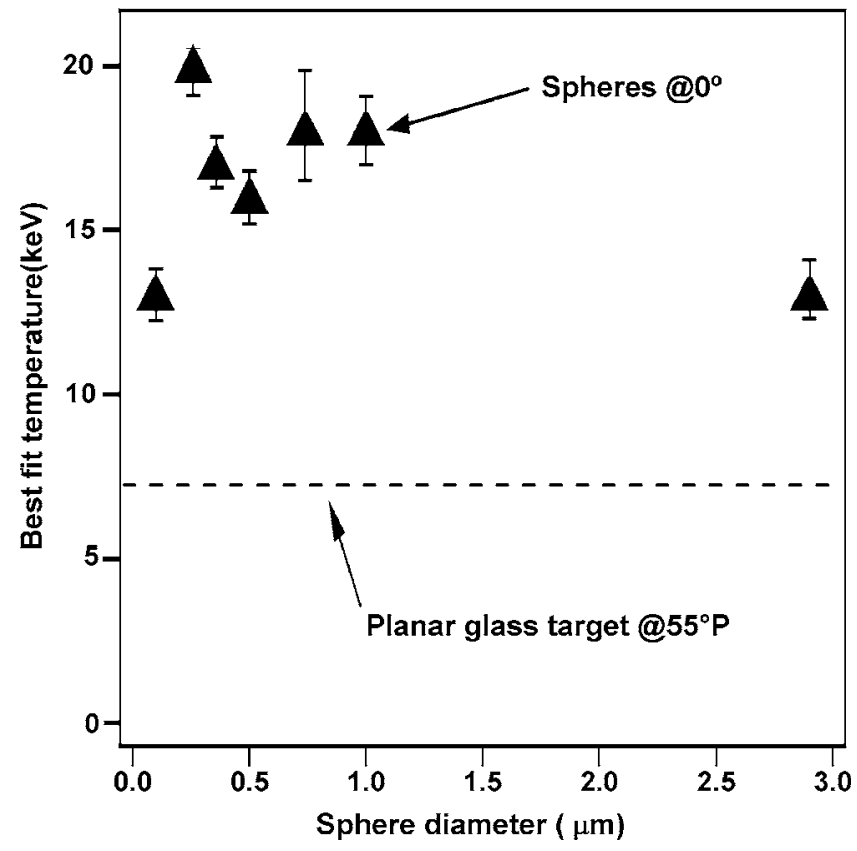

FIG. 8. The hot-electron temperature fit as a function of sphere diameter. The electron temperature peaks weakly near $0.26 \mu \mathrm{m}$.

$100 \mathrm{~nm}, 260 \mathrm{~nm}$, and $1 \mu \mathrm{m}$ spheres. These data are normalized to the yield through the lowest cutoff energy filter. A clear hardening of the $\mathrm{x}$-ray temperature is evident from the slope of the signals when the $100 \mathrm{~nm}$ and $260 \mathrm{~nm}$ spheres are compared. There is less of a difference in the slope of x-ray yield when $260 \mathrm{~nm}$ and $1 \mu \mathrm{m}$ spheres are compared. The hot electron temperature ascertained from these data points for the various sphere sizes is plotted in Fig. 8 as a function of sphere size. The result is that the implied Maxwellian temperature for the hot-electrons also peaked weakly for the $0.26 \mu \mathrm{m}$ spheres. We can conclude that we have observed a sphere size region near $0.26 \mu \mathrm{m}$ where the production of $\mathrm{x}$-rays is optimized. The $\mathrm{x}$-ray yield is larger by a factor of 3 over other sphere sizes, and the temperature of the $\mathrm{x}$-rays is somewhat increased.

\section{DISCUSSION}

Our data indicate that the size of the features on the target have a strong influence on the interaction. We believe that the observed dependence with sphere size is the result of Mie enhancements of the laser field which, accompanied by multipass stochastic heating of the electrons, yield significantly enhanced hard $\mathrm{x}$-ray output and temperature. The angle of incidence scan for planar silica [Fig. 4(b)] indicates that we operate in a vacuum heating regime; the laser interacts with a dense plasma with density scale length smaller than the wavelength. In this situation, the average energy imparted to the electrons is on the order of the ponderomotive energy, which for our conditions is $\sim 3 \mathrm{keV}$. We observe, however, average hot-electron temperatures near $20 \mathrm{keV}$. To accelerate electrons to these energies would require enhancement of the local electric field and/or multiple vacuum heating of the same electrons.
To assess the field generated by the laser at a sphere surface, and the possibility of local field enhancements, we calculated the expected electric field around the spheres assuming a static plasma sphere. Our calculations solve Maxwell's equations in the usual manner for dielectric particles. ${ }^{28}$ The Mie model requires inputting the index of refraction of the sphere, which we calculated using a Drude model. To derive information about the density of the plasma spheres under irradiation, we ran the hydrocode, Hyades, and found that an average ionization state of $Z \approx 3$ occurred during the pulse and the bulk plasma was heated to a temperature on the order of a hundred $\mathrm{eV}$. This implied a plasma density of 14 times critical density (roughly $3 \times 10^{22} \mathrm{~cm}^{-3}$ ).

Using these values for plasma parameters to calculate the index of refraction allows us to calculate the near-field in the vicinity of the sphere. We modeled the field around an isolated sphere using Barber and Hill's ${ }^{29}$ code for spherical particles. To get some insight into the extent of field enhancement that occurs on the surface of the sphere, we plot the absolute square value of the electric field around the spheres, a quantity proportional to the ponderomotive energy of oscillating electrons. This quantity has been normalized to the square of the field in vacuum to indicate how much the presence of the plasma sphere enhances the field. The square of the field distribution around a single sphere normalized in this way is shown in Fig. 9(a). This plot indicates that the field is enhanced by almost a factor of 10 over some surface areas of the sphere.

In order to obtain a closer physical match to our geometry we then explored the electric field resulting from the interaction of many spheres in close proximity. This was done using a code developed by Mackowski et al. ${ }^{30}$ We ran the code with many variations spanning our sphere size range and analyzing different orientations of the spheres, including hexagonal close packed formations and random orientations on a plane. The field distribution around a random array of $260 \mathrm{~nm}$ spheres is illustrated in Fig. 9(b). We find that contact between spheres can lead to even greater field enhancements than are possible with an isolated sphere.

Our final approximation involved solving for the field of a sphere on a surface. This problem is analytically tractable for a perfectly conducting surface. In our case, the substrate surface is ionized by the time the peak of the pulse arrives so we expect the surface to be a good conductor. One convenient technique for calculating the field in this case is the method of images. ${ }^{31}$ The field around a single sphere on a conducting surface is shown in Fig. 9(c). These calculations indicated that the field enhancements in the presence of a substrate do not differ significantly from those of an isolated sphere.

For each of these three cases (an isolated sphere, a single sphere on a perfectly conducting surface, two spheres in close proximity) we found the maximum squared field strength achieved at the highest field point on the surface and plotted this in Fig. 10 as a function of sphere size. For the case of an isolated sphere and a sphere on a substrate, the field peaked at a diameter of approximately $0.1 \mu \mathrm{m}$. In the case of multiple spheres, we analyzed the field around two spheres of identical size which are placed in close proximity 

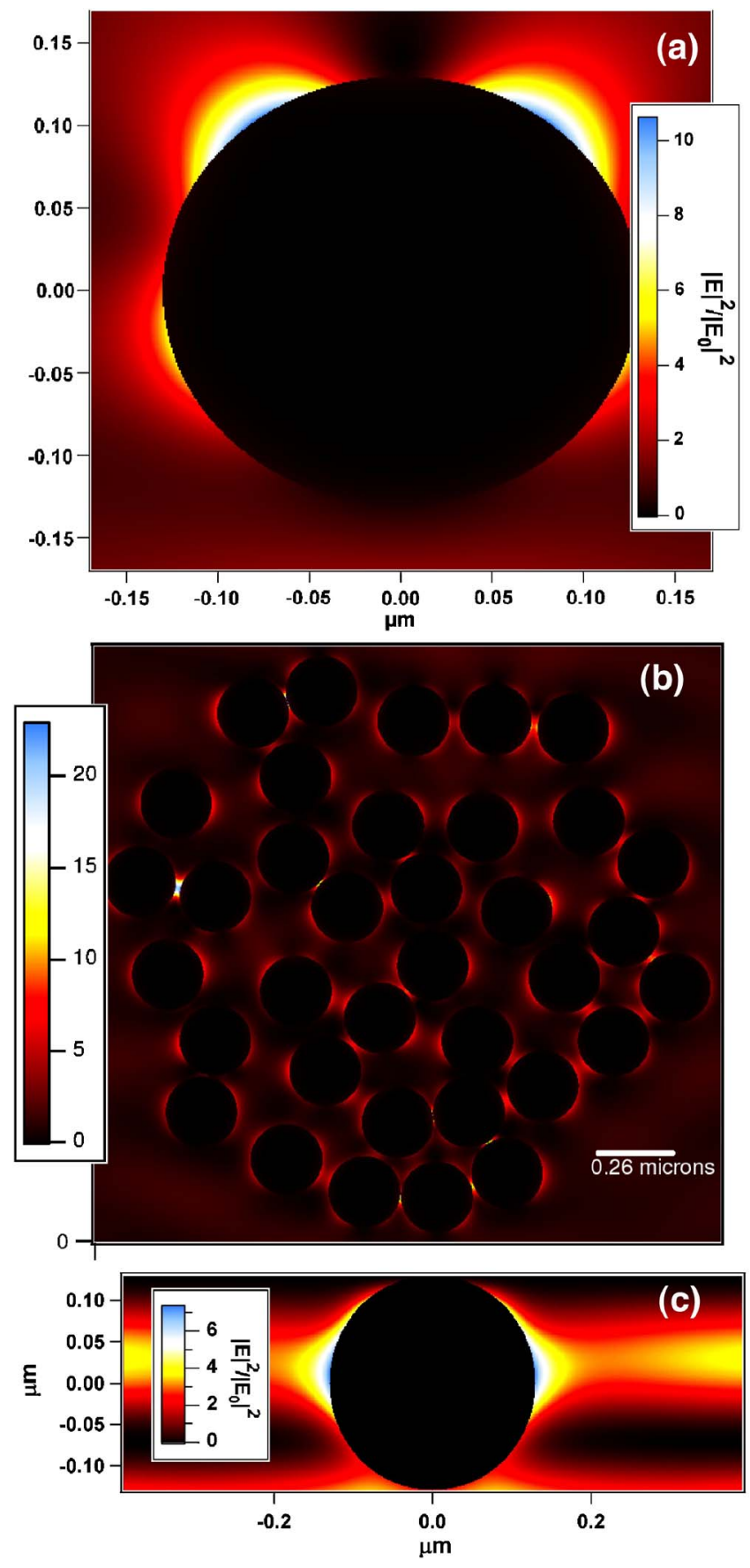

FIG. 9. (Color online) A simulation showing the square of the laser field around (a) a $0.26 \mu \mathrm{m}$ isolated plasma sphere, (b) around randomly positioned $0.26 \mu \mathrm{m}$ spheres and (c) a $0.26 \mu \mathrm{m}$ plasma sphere located on a perfectly conducting plane. The color map is in units of incident laser field strength. In (a), the laser is incident from the top of the figure. In (c), the conducting plane is located across the top of the image, and field enhancements are again evident.

having the line joining their centers in the plane of polarization of the incident field. In this case, the maximum squared field strength was 40 times larger than for isolated spheres. Again the maximum squared field strength peaked, but it was shifted to smaller sphere diameter, $0.07 \mu \mathrm{m}$. In all of these approximations much of the sphere surface can see effective intensity over ten times the vacuum intensity and small areas see 30 times that of the vacuum intensity. This will lead to

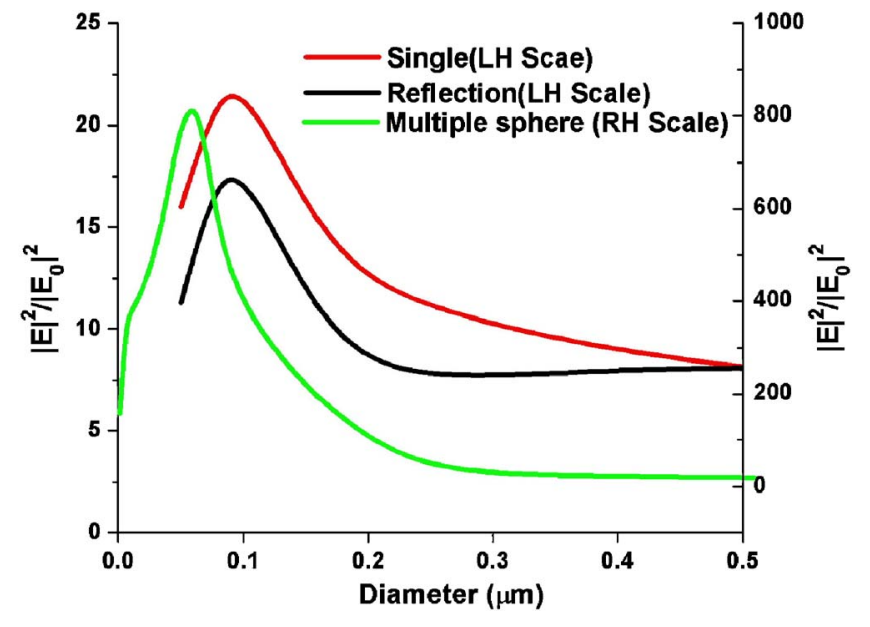

FIG. 10. (Color online) Summary of simulations showing the maximum of the square of the field strength on the sphere surface for an isolated sphere, a single sphere on a perfectly conducting surface, and two spheres in close proximity. The maximum field strength for two spheres is over 40 times larger than for either of the other cases.

greater extraction of electrons and greater acceleration to higher velocity. There is an enhancement of the field with spheres of sizes between 50 and $500 \mathrm{~nm}$ with the largest enhancement with $\sim 100 \mathrm{~nm}$ spheres, close to the size at which we observe the maximum hot electron yield. The fact that we observe the maximum electron enhancement for slightly larger spheres may be a result of the fact that even with the frequency doubled light, some small pre-expansion (a few tens of $\mathrm{nm}$ ) of a target will occur prior to the main pulse arrival. Thus $100 \mathrm{~nm}$ spheres may be so small that they do not survive completely in the rising laser field.

Another possible mechanism for increased electron energy is that of multiple vacuum heating collisions, or stochastic heating. ${ }^{32,33}$ The laser field will accelerate electrons into overdense regions of the plasma sphere where they are shielded from the laser light. During their travel through the sphere the electrons acquire a random phase with respect to the laser. When they re-emerge from the other side, some electrons will be of correct phase to receive a second ponderomotive kick. Under the right circumstances, some electrons can undergo many such vacuum heating passes on each side of the plasma sphere. This multipass heating, first seen in particle-in-cell simulations of Wilks in Ref. 15 and since explored in more detail by Taguchi et al. ${ }^{34}$ and Breizman et $a l .{ }^{33}$ will probably play a role in electron heating from these spheres. In fact, the simulations of Wilks in Ref. 15 showed that micron-scale spheres irradiated by intense $800 \mathrm{~nm}$ pulses should exhibit dramatic hot electron temperature increases from this multipass heating. Quantitative analytic estimates for stochastic heating in this multiple-vacuum-pass model were developed for a sphere much smaller than the wavelength by Breizman et al. ${ }^{33}$ Though they considered spheres much smaller than a wavelength, the arguments relevant to the electron heating can be qualitatively transferred to a sphere of an arbitrary size. Greschik et al. have shown that this multipass stochastic heating will saturate in small clusters due to outer ionization, ${ }^{35}$ however, our wavelength scale spheres are large enough that this saturation should not 
occur. Kishimoto et al. have also considered fast electron ejection from large clusters irradiated at relativistic intensity and have shown that ion expansion plays an important role in the dynamics. ${ }^{36}$ In this case hydrogen was considered, while in our experiment, the higher mass constituents of the clusters will limit this expansion. We therefore believe that the arguments of Briezman, of electron stochastic heating from a rigid ion sphere are appropriate.

It turns out that there are competing effects in this stochastic heating as the sphere size is increased. First, to undergo multiple passes in the plasma sphere, the electron must not be stopped in the plasma by collisions. For stochastic heating to initiate, the vacuum accelerated electrons penetrating the sphere must have sufficient kinetic energy to cross the sphere without being stopped by Coulomb collisions with ions in the plasma. Making an estimate for the collisional mean free path, $\lambda$, of electrons colliding with ions using standard Spitzer-type collision formulas, we can estimate the stopping distance as $\lambda \approx 10^{12} E^{2} / Z n_{i}$, where $E$ is the electron energy in $\mathrm{eV}, Z$ is the ion charge, and $n_{i}$ is the ion density in number $/ \mathrm{cm}^{3}$. For our plasma spheres we estimate $\lambda$ $=0.3 \mu \mathrm{m}$ for a ponderomotive energy of $3 \mathrm{keV}$ (corresponding to our nominal intensity of $2 \times 10^{17} \mathrm{~W} / \mathrm{cm}^{2}$ ). The scattering of electrons inside the larger spheres can thus stop the multiple vacuum heating cycle from initiating for spheres much larger that $300 \mathrm{~nm}$. This will tend to clamp down the production of hot electrons for the larger sized spheres.

On the other hand, with smaller spheres, smaller volume and less efficient stochastic heating (see Ref. 33) lead to lower hot electron production. Our targets consisted of a two-dimensional surface covered with spheres. For a given substrate area, the surface area of spheres will be constant regardless of the sphere size. One of the features of stochastic heating is that the number of electrons participating in the heating per unit area is proportional to the radius of the sphere. This results in fewer electrons being stochastically heated for the smaller spheres. This effect can be visualized in the following way, if one considers the first bunch of electrons which undergo vacuum heating. As they enter the sphere, they no longer shield the sphere and the laser field can accelerate a new bunch of electrons on the next optical cycle. This process continues until the fastest bunch of electrons emerges at the other side of the sphere. At this stage the high density core of the sphere is effectively shielded from the laser field and no further extraction of electrons can occur. This acts to clamp down the number of electrons heated in the smaller size spheres. It seems that these two effects conspire with field enhancements generated by Mie resonances to produce an optimum in hot electron production with spheres of diameter $\sim 260 \mathrm{~nm}$.

To explore the interplay of all of these effects on hot electron production from plasma spheres, particle-in-cell (PIC) simulations of electron heating were conducted using the Virtual Laser Plasma Laboratory (VLPL), relativistic parallelized PIC code of Pukhov et al. ${ }^{37}$ These simulations assumed a $\sim 10$ fs Gaussian pulse with $400 \mathrm{~nm}$ wavelength incident on a plasma sphere with an intensity of $\sim 10^{17} \mathrm{~W} / \mathrm{cm}^{2}$. The plasma sphere was composed of a mixture of carbon and hydrogen ions with an initial electron
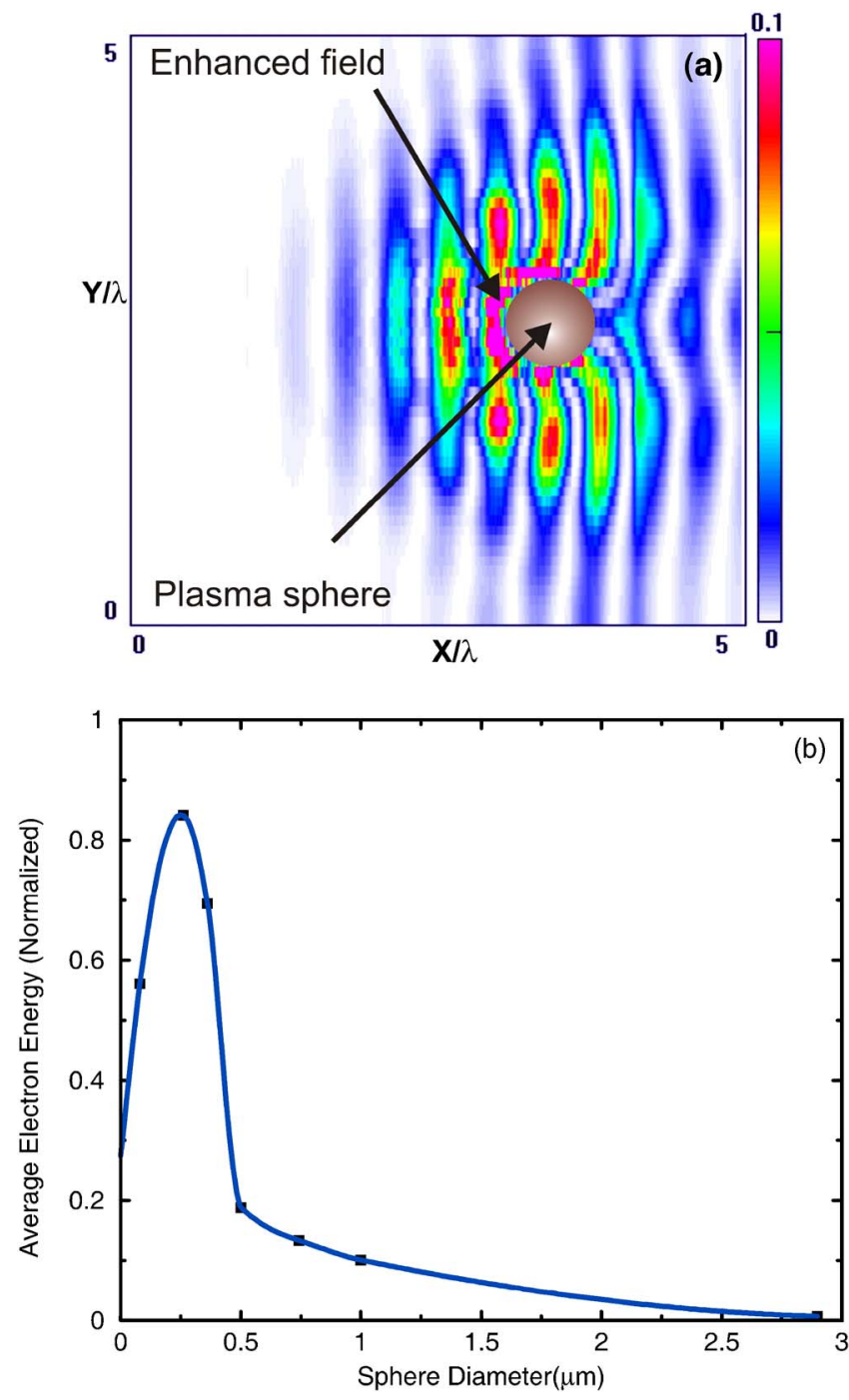

FIG. 11. (Color online) (a) A plot of the square of the electric field during an intense pulse interacting with a $250 \mathrm{~nm}$ plasma sphere as found from the PIC simulations. The enhanced field near the surface of the plasma sphere is readily apparent. (b) A plot of the average hot electron energy in the PIC simulation well after the pulse has passed. The electron energy has been normalized.

density 14 times that of the critical density. The effects of ionizations are also taken into account. The square of the dimensionless field around a plasma sphere calculated with this code during the laser interaction is illustrated in Fig. 11(a). A clear enhancement of the field on the surface of the plasma sphere is evident in this plot. Multipass heating also plays a role in the final electron temperature. Figure 11(b) plots the calculated electron average energy after 40 laser cycles into the interaction. This set of simulations shows that a maximum in the average energy of the hot electrons occurs for plasma spheres of $250 \mathrm{~nm}$ diameter. This represents a remarkable agreement with the trend observed in the experimental data.

\section{CONCLUSION}

In conclusion, we observed that precise control over the microscopic target surface shape allows for control of strong laser field coupling to hot electrons and an enhancement of 
hard $\mathrm{x}$-ray production. This control is demonstrated by the resonance-like behavior in the production of x-rays found for fused silica slides covered by spheres of $0.26 \mu \mathrm{m}$. By choosing spheres with a diameter roughly half that of the laser wavelength, the combination of field enhancements from Mie resonances and efficient stochastic heating of the electrons leads to a substantial increase in hot electron number and an increase in electron temperature. This surface field control is a way to enhance $\mathrm{x}$-ray yield for laser based x-ray sources with a simple alteration to the target.

\section{ACKNOWLEDGMENTS}

We would like to acknowledge the assistance of Boris Breizman and Alex Arefiev for theoretical insight.

This work was supported by the National Science Foundation under Grants Nos. PHY-0456870 and PHY-0456898, the Welch Foundation Grant No. F-1626, the DOE Office of Fusion Energy Science under a Fusion Science Center, the National Nuclear Security Administration under Cooperative Agreement No. DE-FC52-03NA00156. Acknowledgment is made to the Donors of the American Chemical Society Petroleum Research Fund for partial support of this research.

${ }^{1}$ A. Rousse, C. Rischel, S. Fourmaux et al., Nature (London) 410, 65 (2001).

${ }^{2}$ C. W. Siders, A. Cavalleri, K. Sokolowski-Tinten et al., Science 286, 1340 (1999).

${ }^{3}$ R. L. McCrory, S. P. Regan, S. J. Loucks et al., Nucl. Fusion 45, S283 (2005).

${ }^{4} \mathrm{P}$. Gibbon, Short-Pulse Laser-Plasma Interactions With Matter: An Introduction (Imperial College Press, London, 2005), pp. 169-179.

${ }^{5}$ W. L. Kruer, The Physics of Laser Plasma Interactions (Westview, Boulder, 2003).

${ }^{6}$ F. Brunel, Phys. Rev. Lett. 59, 52 (1987).

${ }^{7}$ M. M. Murnane, H. C Kapteyn, S. P Gordon, J. Bokor, E. N. Glytsis, and R. W. Falcone, Appl. Phys. Lett. 62, 1068 (1993).

${ }^{8}$ S. P. Gordon, A. Sullivan, H. Hamster, and R. W. Falcone, Opt. Lett. 19, 484 (1994).

${ }^{9}$ G. Kulcsar, D. AlMawlawi, F. W. Budnik, P. R. Herman, M. Moskovits, L. Zhao, and R. S. Marjoribanks, Phys. Rev. Lett. 84, 5149 (2000).

${ }^{10}$ P. P. Rajeev, S. Banerjee, A. S. Sandhu, R. C. Issac, L. C. Tribedi, and G. Ravindra Kumar, Phys. Rev. A 65, 052903 (2002).
${ }^{11}$ P. P. Rajeev, P. Taneja, P. Ayyub, A. S. Sandhu, and G. Ravindra Kumar, Phys. Rev. Lett. 90, 115002 (2003).

${ }^{12}$ P. P. Rajeev and G. Ravindra Kumar, Opt. Commun. 222, 9 (2003).

${ }^{13}$ S. A. Gavrilov, V. M. Gordienko, A. B. Savel'ev, and R. V. VolKov, Laser Part. Beams 22, 301 (2004).

${ }^{14}$ G. Mie, Ann. Phys. 25, 377 (1908).

${ }^{15}$ T. D. Donnelly, M. Rust, I. Weiner et al., J. Phys. B 34, L313 (2001).

${ }^{16}$ E. T. Gumbrell, A. J. Comley, M. H. R. Hutchinson, and R. A. Smith, Phys. Plasmas 8, 1329 (2001).

${ }^{17}$ E. Parra, S. J. McNaught, J. Fan, and H. M. Milchberg, Appl. Phys. A A77, 317 (2003).

${ }^{18}$ Y. T. Li, J. Zhang, Z. M. Sheng, H. Teng, T. J. Liang, X. Y. Peng, X. Lu, Y. J. Li, and X. W. Tang, Phys. Rev. Lett. 90, 165002 (2003).

${ }^{19}$ X. Y. Peng, J. Zhang, J. Zheng et al., Phys. Rev. E 74, 036405 (2006).

${ }^{20}$ D. Riley, J. J. Angulo-Gareta, F. Y. Khattak, M. J. Lamb, P. S. Foster, E. J. Divall, C. J. Hooker, A. J. Langley, R. J. Clarke, and D. Neely, Phys. Rev. E 71, 016406 (2005).

${ }^{21}$ D. R. Symes, A. J. Comley, and R. A. Smith, Phys. Rev. Lett. 93, 145004 (2004).

${ }^{22}$ T. Ditmire, S. Bless, G. Dyer, A. Edens, W. Grigsby, G. Hays, K. Madison, A. Maltsev, J. Colvin, M. J. Edwards, R. W. Lee, P. Patel, D. Price, B. A. Remington, R. Sheppherd, A. Wootton, J. Zweiback, E. Liang, and K. A. Kielty, Radiat. Phys. Chem. 70, 535 (2004).

${ }^{23}$ R. Micheletto, H. Fukuda, and M. Ohtsu, Langmuir 11, 3333 (1995).

${ }^{24}$ Y. B. Zel'dovich and Y. P. Raizer, Physics of Shock Waves and High Temperature Hydrodynamic Phenomena (Academic, New York, 1968), p. 258.

${ }^{25}$ P. Gibbon, Phys. Rev. Lett. 68, 1535 (1992).

${ }^{26}$ H. Sumeruk, T. Ditmire, T. D. Donnelly et al., Bull. Am. Phys. Soc. 50, \#QP1.083 (2005).

${ }^{27}$ G. H. McCall, J. Phys. D 15, 823 (1982).

${ }^{28}$ M. Born and E. Wolf, Principles of Optics (Pergamon, Oxford, 1980), p. 635.

${ }^{29}$ P. W. Barber and S. Hill, Light Scattering by Particles: Computational Methods (World Scientific, Singapore, 1990).

${ }^{30}$ D. W. Mackowski and M. I. Mishchenko, J. Opt. Soc. Am. A 13, 2266 (1996).

${ }^{31}$ B. R. Johnson, J. Opt. Soc. Am. A 9, 1341 (1992).

${ }^{32}$ T. M. Antonsen, T. T. Ayush Gupta, J. Palastro, and H. M. Milchberg, Phys. Plasmas 12, 056703 (2005).

${ }^{33}$ B. N. Breizman, A. V. A. Mykhailo, and V. Fomys'kyi, Phys. Plasmas 12, 056706 (2005).

${ }^{34}$ T. Taguchi, T. M. Antonsen, and H. M. Milchberg, Phys. Rev. Lett. 92, 205003 (2004).

${ }^{35}$ F. Greschik, L. Arndt, and H.-J. Kull, Europhys. Lett. 72, 376 (2005).

${ }^{36}$ Y. Kishimoto, T. Masaki, and T. Tajima, Phys. Plasmas 9, 589 (2002).

${ }^{37}$ A. Pukhov, J. Plasma Phys. 61, 425 (1999). 